\title{
ASSESSMENT OF SUSCEPTIBILITY TO LIQUEFACTION OF SATURATED ROAD EMBANKMENT SUBJECTED TO DYNAMIC LOADS
}

\author{
ANNA BOROWIEC \\ Faculty of Mining and Geoengineering, AGH University of Science and Technology
}

\section{KRZYSZTOF MACIEJEWSKI}

Faculty of Civil Engineering and Architecture, Kielce University of Technology

\begin{abstract}
Liquefaction has always been intensely studied in parts of the world where earthquakes occur. However, the seismic activity is not the only possible cause of this phenomenon. It may in fact be triggered by some human activities, such as constructing and mining or by rail and road transport.

In the paper a road embankment built across a shallow water reservoir is analyzed in terms of susceptibility to liquefaction. Two types of dynamic loadings are considered: first corresponding to an operation of a vibratory roller and second to an earthquake.

In order to evaluate a susceptibility of soil to liquefaction, a factor of safety against triggering of liquefaction is used $\left(F S_{\text {Triggering }}\right)$. It is defined as a ratio of vertical effective stresses to the shear stresses both varying with time. For the structure considered both stresses are obtained using finite element method program, here Plaxis 2D. The plastic behavior of the cohesionless soils is modeled by means of Hardening Soil (HS) constitutive relationship, implemented in Plaxis software.

As the stress tensor varies with time during dynamic excitation, the $F S_{\text {Triggering }}$ has to be calculated for some particular moment of time when liquefaction is most likely to occur. For the purposes of this paper it is named a critical time and established for reference point at which the pore pressures were traced in time. As a result a factor of safety distribution throughout embankment is generated.

For the modeled structure, cyclic point loads (i.e., vibrating roller) present higher risk than earthquake of magnitude 5.4. Explanation why considered structure is less susceptible to earthquake than typical dam could lay in stabilizing and damping influence of water, acting here on both sides of the slope.

Analogical procedure is applied to assess liquefaction susceptibility of the road embankment considered but under earthquake excitation. Only the higher water table is considered as it is the most unfavorable.

Additionally the modified factor of safety is introduced, where the dynamic shear stress component is obtained at a time step when its magnitude is the highest - not necessarily at the same time step when the pore pressure reaches its peak (i.e., critical time). This procedure provides a greater margin of safety as the computed factors of safety are smaller.

Method introduced in the paper presents a clear and easy way to locate liquefied zones and estimate liquefaction susceptibility of the subsoil - not only in the road embankment.
\end{abstract}

Key words: liquefaction susceptibility, soil dynamic, seismic lads, vibrations propagation in soil

\section{INTRODUCTION}

Liquefaction is a phenomenon most frequently observed in medium and fine grained saturated cohesionless soils subjected to dynamic loading. Due to rapid cyclic changes, the free drainage cannot occur and the overburden pressure is no longer sustained by the soil skeleton mainly but it is hugely transferred to the pore water. As a result, an increase of pore pressure is observed along with the reduction of shear strength and the capacity of the soil to sustain loading.
Liquefaction may lead to uneven and significant settlements of buildings, failures of soil structures such as road embankments and dams, landslides and other hazardous phenomena. Despite such an important role liquefaction still lacks quick and simple assessment that can be used in geotechnical design.

\section{CASE STUDY}

The literature mentions a case of failure of road embankment across the Ackerman Lake induced by six 
Vibroseis vehicles conducting a geophysical survey [1]. A Vibroseis is used to excite the ground via a vibrating metal plate moved by an eccentric weight. As the trucks were spread out over $74 \mathrm{~m}$ along embankment, they were set to operate in phase for 8 seconds. The excitation frequency changed linearly from 8 to $58 \mathrm{~Hz}$ to give a sum of 264 cycles. Soon after the equipment was activated the saturated soil liquefied sinking five trucks. The failure was rapid enough to cause $4.5 \mathrm{~m}$ wave on the waters of Ackerman Lake.

In the paper a similar case is examined. A road embankment built across a shallow water reservoir is analyzed in terms of susceptibility to liquefaction. Two types of dynamic loadings are considered: first corresponding to an operation of a vibratory roller and second to an earthquake.

\subsection{GEOMETRY}

The embankment is three meter high above the reservoir bed with a ten meter wide crest. The structure has a slope of $1.5 \mathrm{H}: 1 \mathrm{~V}$ with approx. $0.8 \mathrm{~m}$ facing of clayey soil. The embankments core is mainly made of fluvial medium density, mediumgrained sands topped by two layers of glacial dense sands (see Fig. 1). The surface of water is set to $1 \mathrm{~m}$ and $2 \mathrm{~m}$ above the reservoir bottom giving the Water Table Level (WTL) at $5 \mathrm{~m}$ and at $6 \mathrm{~m}$, respectively (compare Fig. 5 and Fig. 6).

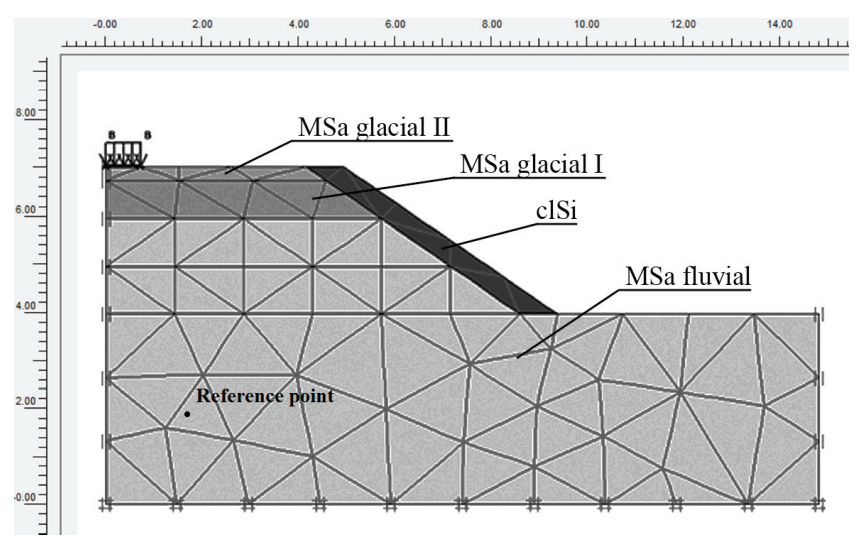

Fig. 1. Model geometry and finite element mesh

\subsection{FEM MODEL IN PLAXIS 2D}

Plaxis 2D is a program based on Biot's theory of dynamic consolidation with a possibility to use advanced elastoplastic constitutive relationships. The 15-node Finite Elements are applied to generate the coarse mesh with (in this example) the average element size smaller than one meter.

Such coarseness of the mesh is chosen to save computational time and the FE dimension is selected with respect to wavelength. Wavelength can be derived from well-known relationship between velocity of the propagating wave and the vibration frequency of the source [2]. For the kind of soil considered (medium sands), propagating wave velocities diverge between $160 \mathrm{~m} / \mathrm{s}$ and $220 \mathrm{~m} / \mathrm{s}$ for shear and longitudinal wave, respectively. With vibratory roller frequency of $55 \mathrm{~Hz}$ this gives the average wavelength of $4 \mathrm{~m}$. For the non-stationary dynamic excitations like earthquakes, frequencies vary with time and are higher (ranges between 70-140 Hz) [3] causing that the length of the propagating waves is smaller, but still above $1.0 \mathrm{~m}$. Thus the size of the common element less than $1.0 \mathrm{~m}$ is sufficient for this dynamic problem.

To prevent the reflection and refraction of propagating waves, viscous boundary is introduced.

The full geometry is used for modeling soil behavior during earthquake while for cyclic point load only a half of the embankment is considered. Thus, both Plane Strain and Axisymmetry are employed.

\subsection{MATERIALS}

The plastic behavior of the cohesion less soils is modeled by means of Hardening Soil (HS) constitutive relationship, implemented in Plaxis software.

The Hardening Soil (HS) model is an advanced model for simulating the behavior of different types of soil, both soft soils and stiff soils [4]. When subjected to primary deviatoric loading, soil shows a decreasing stiffness and simultaneously irreversible plastic strains develop. In drained triaxial test, the observed relationship between the axial strain and the deviatoric stress can be well approximated by a hyperbola (well-known hyperbolic model). The Hardening Soil model is based on this approach, however supersedes the hyperbolic model by far. Firstly by using the theory of plasticity rather than the theory of elasticity, secondly by including soil dilatancy and thirdly by introducing a yield cap. Representation of yield surface of the Hardening Soil model in principal stress space for cohesionless soil is demonstrated in Fig. 2. The advantage of HS model is not only the use of hyperbolic stress-strain curve but also the control of stress level dependence. In contrast to an elastic perfectly-plastic model, the yield surface of a hardening plasticity model is not fixed in principal stress space, but it can expand due to plastic straining. Distinction can be 
made between two main types of hardening, namely shear hardening and compression hardening. Shear hardening is used to model irreversible strains due to primary deviatoric loading and compression hardening - to irreversible plastic strains due to primary compression in oedometer (and isotropic) loading. Both types of hardening are contained in the present model.

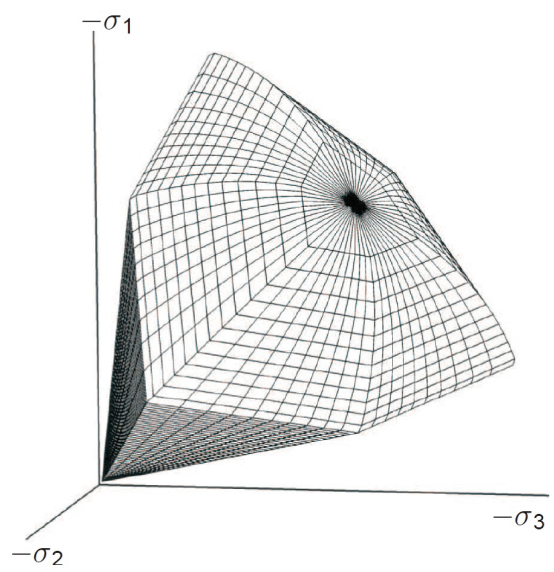

Fig. 2. Yield surface of the HS model
As a basic feature of HS model is stress dependence of soil stiffness, the introduction of cap surface is needed. Without such a cap it would not be possible to formulate a model with independent input of both $E_{50}^{\text {ref }}$, $E_{\text {oed }}^{\text {ref }}$. The triaxial modulus $E_{50}^{\text {ref }}$ is responsible for the shear yield surface and the oedometer modulus $E_{\text {oed }}^{\text {ref }}$ for the cap. In fact, the first one controls the magnitude of the plastic strains that are associated with shear yield surface, while the second is used to manage the magnitude of the plastic strains that originate from yield cap. All characteristics of the model are gathered in Table 1.

Soil parameters for medium sands and clayey silt used in the example are accordingly modified but based on those found in the literature [5]. Material properties along with the model parameters for each layer are specified in Table 2 .

\subsection{TYPES OF LOADING}

In the first case a cyclic load simulating an operation of vibratory roller is introduced. The load posi-

Hardening soil model parameters

\begin{tabular}{|c|c|c|}
\hline Symbol & Description, definition & Expressing \\
\hline$m$ & power for stress-level dependence of stiffness & hyperbolic model \\
\hline$E_{50}^{\text {ref }}$ & secant stiffness in drained standard triaxial test & plastic straining due to primary deviatoric loading, \\
\hline$E_{\text {oed }}^{\text {ref }}$ & tangent stiffness for primary oedometer loading & plastic straining due to primary compression \\
\hline$E_{u r}^{\text {ref }}, v$ & $E_{u r}^{\text {ref }}=3 E_{50}^{\text {ref }}$ & elastic unloading/reloading stiffness and Poisson's ratio \\
\hline$c, \varphi, \psi$ & $\begin{array}{c}\text { failure parameters according to } \\
\text { Mohr-Coulomb }(\mathrm{M}-\mathrm{C}) \text { criteria }\end{array}$ & cohesion, angle of internal friction, dilatancy \\
\hline
\end{tabular}

Table 2

Soil properties

\begin{tabular}{|c|c|c|c|c|c|}
\hline Soil & $\begin{array}{c}\text { MSa } \\
\text { fluvial }\end{array}$ & $\begin{array}{c}\text { MSa } \\
\text { glacial I }\end{array}$ & $\begin{array}{c}\text { MSa } \\
\text { glacial II }\end{array}$ & $\mathrm{clSi}$ & Units \\
\hline Soil model & HS & $\mathrm{HS}$ & HS & $\mathrm{M}-\mathrm{C}$ & - \\
\hline$D_{r}$ & 0.6 & 0.7 & 0.8 & - & - \\
\hline$\gamma$ & 16 & 17 & 18 & 16 & $\mathrm{kN} / \mathrm{m}^{3}$ \\
\hline$\gamma^{\prime}$ & 18 & 19.5 & 20 & 20 & $\mathrm{kN} / \mathrm{m}^{3}$ \\
\hline$c$ & 0.1 & 0.1 & 0.1 & 5 & $\mathrm{kPa}$ \\
\hline$\varphi$ & $35^{\circ}$ & $37^{\circ}$ & $40^{\circ}$ & $24^{\circ}$ & - \\
\hline$\psi$ & $5^{\circ}$ & $7^{\circ}$ & $10^{\circ}$ & - & - \\
\hline$v$ & 0.2 & 0.2 & 0.2 & 0.35 & - \\
\hline$m$ & 0.5 & 0.5 & 0.5 & - & - \\
\hline$E$ & - & - & - & 2000 & $\mathrm{kPa}$ \\
\hline$E_{50}^{\mathrm{ref}}$ & 35.000 & 40.000 & 45.000 & - & $\mathrm{kPa}$ \\
\hline$E_{\text {oed }}^{\text {ref }}$ & 35.000 & 40.000 & 45.000 & - & $\mathrm{kPa}$ \\
\hline$E_{u r}^{\mathrm{ref}}$ & 10.500 & 12.000 & 13.500 & - & $\mathrm{kPa}$ \\
\hline
\end{tabular}


tioned at the center of the embankment crest interacts with the soil through a stiff plate element $1.5 \mathrm{~m}$ wide. The loading itself has two components: static with a magnitude of $119 \mathrm{kN} / \mathrm{m}$ and harmonic with an amplitude of $350 \mathrm{kN} / \mathrm{m}$ and frequency of $55 \mathrm{~Hz}$.

The second case of load is an earthquake excitation of magnitude 5.4 on the Open-Ended Richter Scale. Epicenter distance is $5.0 \mathrm{~km}$ and peak acceleration $240 \mathrm{~cm} / \mathrm{s}^{2}$. In Plaxis 2D, like in most FEM programs, earthquake is modeled by imposing prescribed displacements at the bottom boundary, calculated by program itself from accelerogram shown in Fig. 3. These data are downloaded from United States Geological Survey and were recorded on 28th of February 1990 by station no. 0656 .

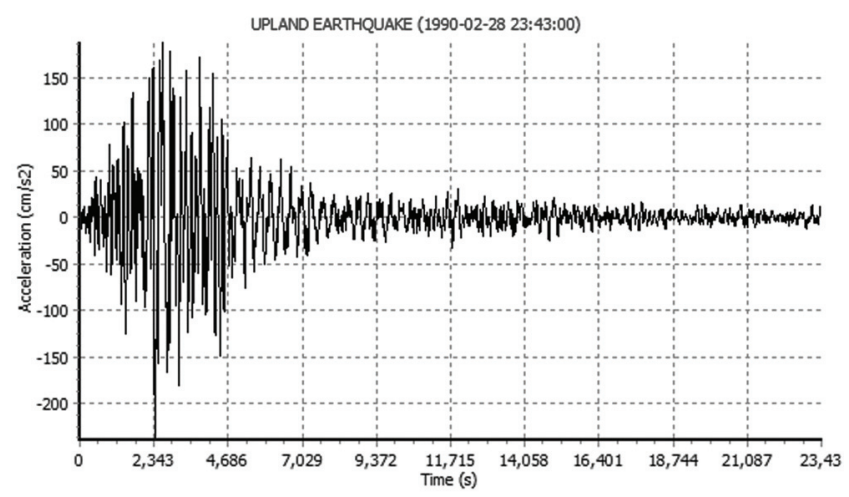

Fig. 3. Earthquake accelerogram

\section{FACTOR OF SAFETY CALCULATION}

In the issue considered, a simple and practical approach is to use a factor of safety against triggering of liquefaction. It can be defined as

$$
F S_{\text {Triggering }}=\frac{s_{u}(\text { yield })}{\tau_{\text {driving }}+\tau_{\text {dynamic }}+\tau_{\text {other }}}
$$

where:\

$s_{u}($ yield $)$ - undrainedyield shear strength,

$\tau_{\text {driving }}-$ static shear stress,

$\tau_{\text {dynamic }}-$ shear stresses caused by dynamic loading,

$\tau_{\text {other }}$ - other shear stresses.

The undrained yield shear strength can be derived from the yield strength ratio expressed as

$$
\frac{s_{u}(\text { yield })}{\sigma_{v 0}^{\prime}}=\tan \phi_{y}
$$

where

$\sigma_{v 0}^{\prime}-$ vertical or normal effective stress,

$\varphi_{y}-$ mobilized yield friction angle.
Based on the field test regarding relationship between SPT blow counts and CPT tip resistance and the yield strength ratio, the mobilized yield friction angle of the analyzed soils (MSa fluvial) can be determined as $\varphi_{y}=13^{\circ}$ [6]. It can also be retrieved from phi-c reduction procedure during numerical analysis.

Containing normal effective stresses, from rewritten equation (2) it is possible to compute the yield shear strength $s_{u}$ (yield). The $F S_{\text {Triggering }}$ is therefore dependent only on two unknown variables: shear stress $\tau_{\text {dynamic }}$ and vertical or normal effective stress $\sigma_{v 0}^{\prime}$ and can be written at point $P_{x, y}$ as

$$
F S_{\text {Triggering }}\left(P_{x, y}\right)=\frac{\tan \phi_{y} \sigma_{v 0}^{\prime}\left(P_{x, y}\right)}{\tau_{\text {dynamic }}\left(P_{x, y}\right)}
$$

where

$\sigma_{v 0}^{\prime}\left(P_{x, y}\right)$ - vertical effective stress at point $P_{x, y}$,

$\tau_{\text {dynamic }}\left(P_{x, y}\right)$ - sum of dynamic and static shear stresses at point $P_{x, y}$.

For the structure considered both stresses are obtained using finite element method program, here Plaxis 2D.

\section{CRITICAL TIME DETERMINATION}

As the stress tensor varies with time during dynamic excitation, the $F S_{\text {Triggering }}$ has to be calculated for some particular moment of time when liquefaction is most likely to occur. For the purposes of this paper it is named a critical time and established for reference point at which the pore pressures were traced in time. The reference point is located in one-third of the embankment height, close to its core (see Fig. 1). The critical time takes place when pore pressure reaches its peak value. In the case of cyclic point load (vibratory roller) the critical time is established as 0.08 second.

Finding critical time for earthquake excitation is much more demanding, though. It is well known that liquefaction can occur not only during the earthquake but also after it finishes.

In the modeled embankment pore pressure is building up at the beginning and reaches its peak value for the first time in the 8th second of excitation. Then, after showing some drop, it grows again. Pore pressure changes in time during earthquake at reference point are shown in Fig. 4. In order to select proper critical time, a factor of safety distribution was generated - in the way described in the following paragraph - for the 8th and the 18th second of excita- 


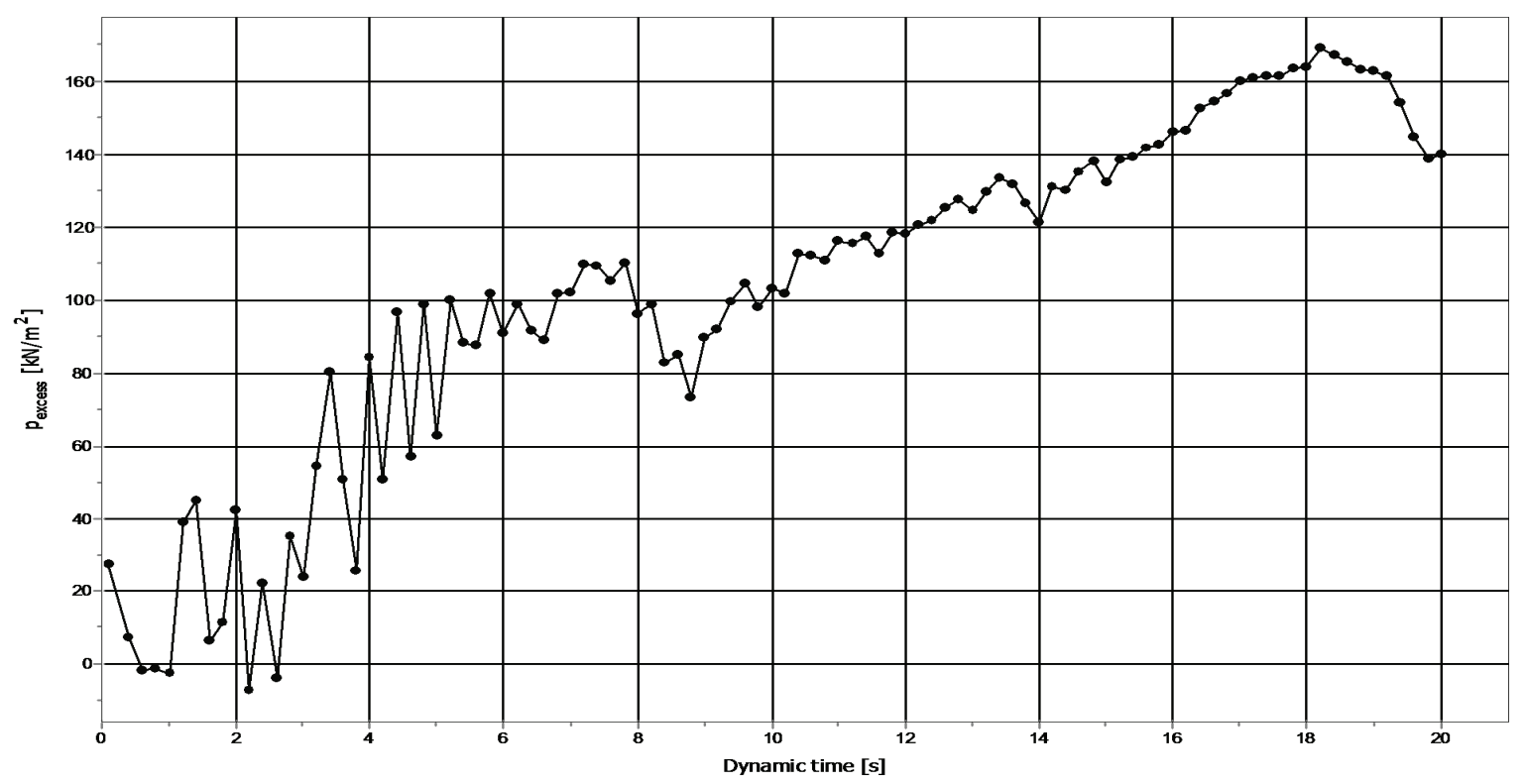

Fig. 4. Pore pressure changes with time during earthquake at reference point

tion, i.e., when the first peak occurs and after the earthquake is finished. That was quite obvious as the pore pressure always accumulates during earthquakes reaching its maximum values at the end or even after the excitation finishes.

\section{RESULTS FOR CYCLIC LOAD}

Factor of safety distribution inside embankment is generated on the basis of vertical effective stress and sum of dynamic and static shear stresses. Both stresses are taken from cross sections put up along the embankment in $1.0 \mathrm{~m}$ spacing, at the critical time.

The allocation of $F S_{\text {Triggering }}$ for cyclic load depending on water table is shown in Fig. 5 and Fig. 6. The liquefaction occurs when $F S_{\text {Triggering }}$ is lower than 1.0 and the areas susceptible to it are bold outlined.

In the case of cyclic point loading (regardless of the water table level) two regions of the embankment are especially exposed to liquefaction. As long as these two regions are separated the overall stability should be kept. On the other hand, very low values of $F S_{\text {Triggering, }}$ at the toe of the slope indicate the loss of the local stability which can also lead to total failure of the structure.

Embankment sections lying straight below the dynamic loading are the second most exposed to liquefying. Area prone to liquefaction observed in Fig. 5 and Fig. 6 spreads also above the free water surface which takes place due to suction in these glacial Medium Sands (causing the full saturation of pores). A map of those areas (created from slices of $1.0 \mathrm{~m}$ width around cross sections mentioned), with calculated $F S_{\text {Triggering, }}$ gives qualitative and quantitative assessment of liquefaction in a saturated road embankment.

\section{RANGE OF DYNAMIC EFFECTS}

In order to check the range of effects of dynamic actions, twelve points distributed evenly over the embankment are selected. They are lying at a similar depth spreading along the width of embankment: first point - beneath the load and last one - near the right border of the model. At these points shear stress $\tau_{x y}$ changes over time are examined. Figure 7 shows relationships of $\tau_{x y}$ versus time and location of the points.

In all the above graphs the delay of signal in space is clearly visible. It arrives at point " $\mathrm{K}$ " even $0.22 \mathrm{~s}$ after the beginning of the excitations. The point " $\mathrm{K}$ " is at a distant of $10 \mathrm{~m}$ from "C", which gives the velocity of traveling signal equal to $45 \mathrm{~m} / \mathrm{s}$. This value is almost 3 times smaller than the shear wave velocity of ideally elastic material (see Section 2.2) proving large damping in space.

It can be seen for point "E" that the amplitude of shear stresses increases with time, but after some period stabilizes at a certain level with a minor attenuation (damping in time). Stress changes with time are also getting smaller with distance from the source of excitation. For the "J" point amplitude of shear stress $\tau_{x y}$ decreases to values smaller than $1 \mathrm{kPa}$. It can therefore be assumed that the range of dynamic influences ends at the lower edge of the escarpment, or just before it. 


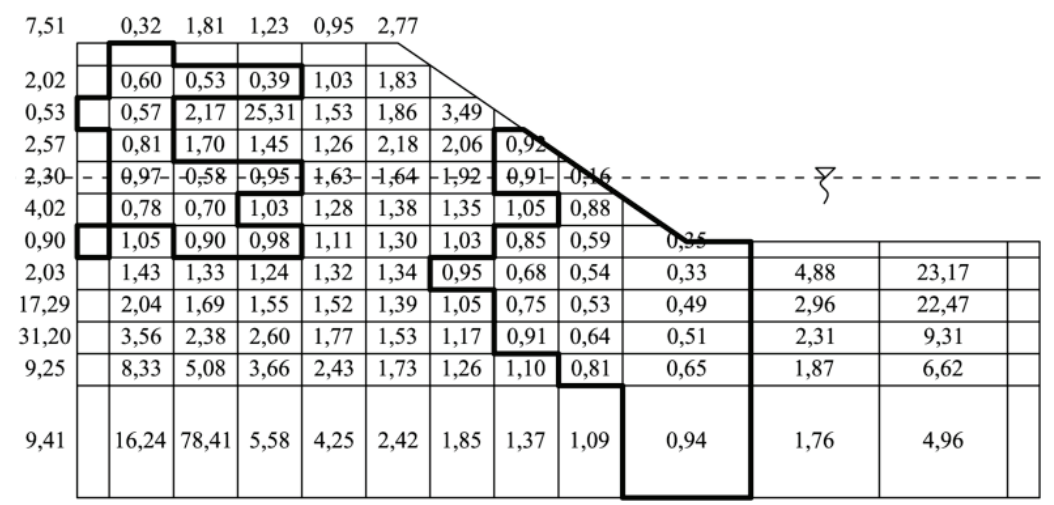

Fig. 5. Factor of safety distribution inside embankment for cyclic load (WTL at $5 \mathrm{~m}$ )

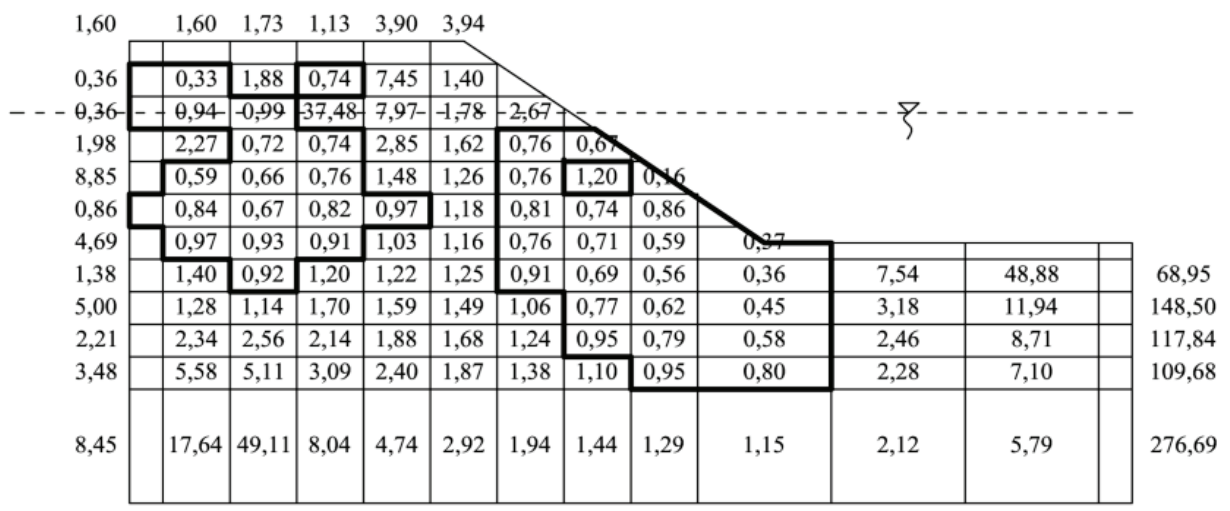

Fig. 6. Factor of safety distribution inside embankment for cyclic load (WTL at $6 \mathrm{~m}$ )

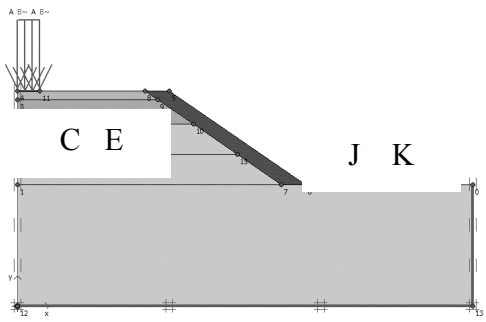

Location of selected points

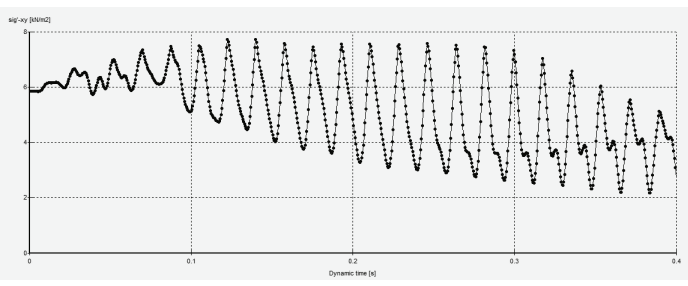

"E” Point

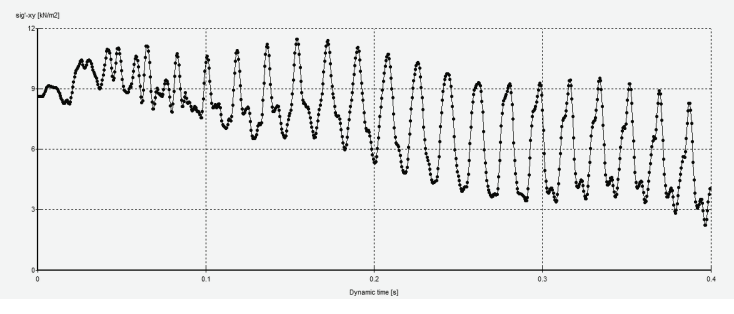

"C" Point

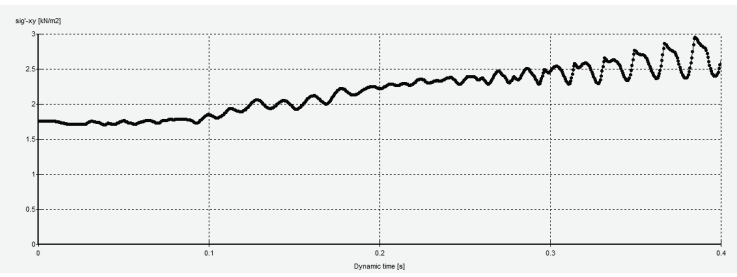

"J" Point

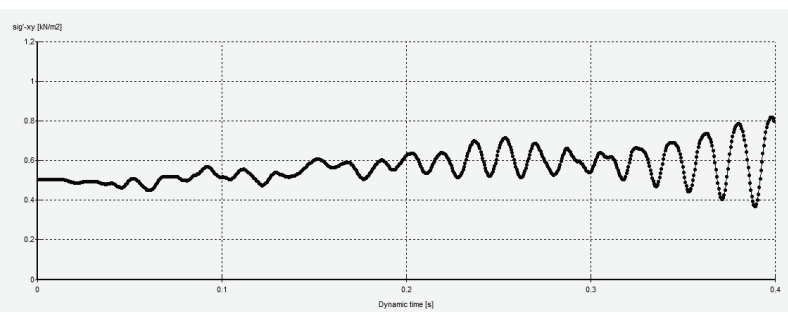

"K" Point

Fig. 7. Shear stresses over time at selected points 


\section{MODIFIED FACTOR OF SAFETY}

As stated before in equation (3), the factor of safety against triggering liquefaction is defined as a ratio of vertical effective stress $\sigma_{v 0}^{\prime}$ to the shear stresses $\tau_{d y n}$ both varying with time. During excitation, different combinations of these two stresses can occur, resulting in different values of the factor of safety. To accommodate this fact, a modified factor of safety is introduced, where the dynamic shear stress component is obtained at a time step when its magnitude is the highest - not necessarily at the same time step when the pore pressure reaches its peak (i.e., critical time). This procedure provides a greater margin of safety as the computed factors of safety are smaller. (shear stresses $\tau_{x y}$ obtained for pore pressure peaks) at the selected points are shown in Table 3 . All these values were used to calculate both factors of safety. Table 3 shows also the coordinates of the centers of slices to which structure was divided in order to create maps of $F S_{\text {Triggering }}$ distribution.

\section{RESULTS FOR EARTHQUAKE EXCITATION}

Analogous procedure is applied to assess liquefaction susceptibility of the road embankment considered but under earthquake excitation. Only the higher water table is considered as it is the most unfavorable.

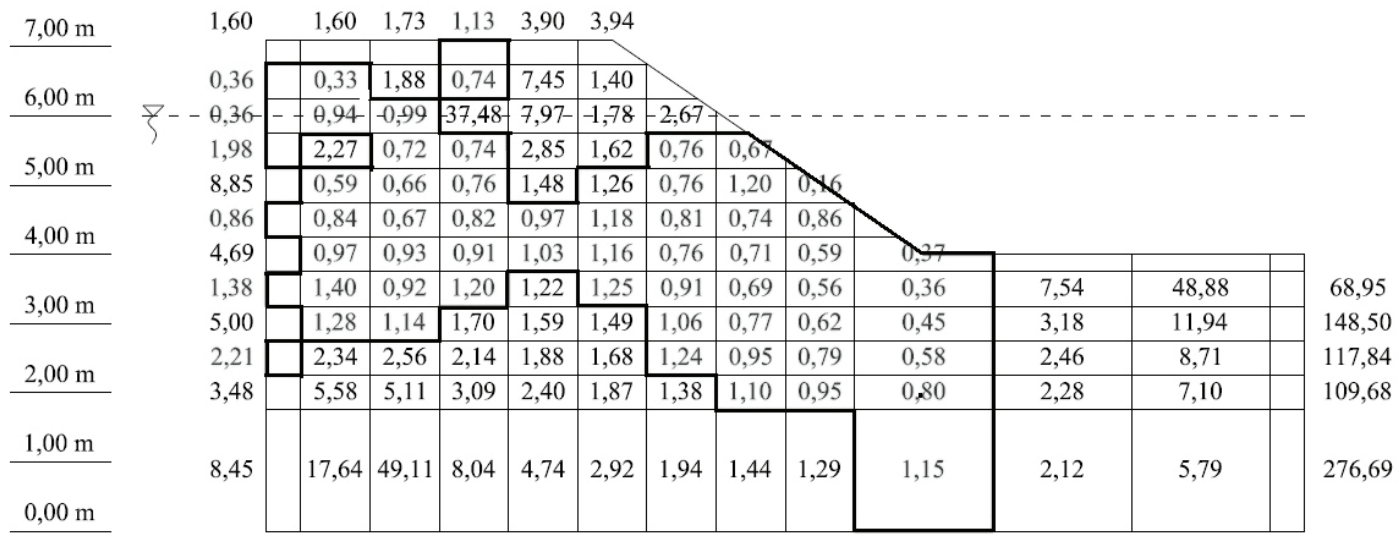

Fig. 8. Modified factor of safety distribution inside embankment for cyclic load (WTL at $6 \mathrm{~m}$ )

Table 3

Ratio of $\tau_{\max }$ to $\tau_{\mathrm{dyn}}$

\begin{tabular}{|c|c|c|c|c|c|c|c|c|c|c|c|}
\hline Point & A & B & C & D & E & F & G & H & I & G & Units \\
\hline$X$ & 0.0 & 1.0 & 2.0 & 3.0 & 4.0 & 5.0 & 6.0 & 7.0 & 8.0 & 9.5 & {$[\mathrm{~m}]$} \\
\hline$Y$ & 4.5 & 4.5 & 4.5 & 4.5 & 4.5 & 4.5 & 4.5 & 4.5 & 3.5 & 3.5 & {$[\mathrm{~m}]$} \\
\hline$\tau_{\max }$ & 20.93 & 14.57 & 11.45 & 11.06 & 7.71 & 7.45 & 6.58 & 4.22 & 5.71 & 2.93 & $\mathrm{kPa}$ \\
\hline$\tau_{\text {dyn }}$ & 8.16 & 8.32 & 9.26 & 8.04 & 6.51 & 5.76 & 5.00 & 3.26 & 4.54 & 2.41 & $\mathrm{kPa}$ \\
\hline$\tau_{\max } / \tau_{\text {dyn }}$ & 2.56 & 1.75 & 1.24 & 1.38 & 1.18 & 1.29 & 1.32 & 1.29 & 1.26 & 1.22 & - \\
\hline
\end{tabular}

It should be noted that also the slope of the embankment is threatened (see Fig. 8). The reason for this is an abiding high level of shear stresses while at the same time disparities between effective stresses and pore pressures are growing. This leads to a significant enlargement of the liquefied zones and as a result to the general weakening or failure of the structure.

The maximum values of shear stresses $\tau_{\max }$ $\left(\tau_{x y_{-} \max }\right)$ in time and the previously read values of $\tau_{\mathrm{dyn}}$,
Factors of Safety at 8th second do not indicate any possible liquefaction in any area so its distribution is not included in this paper. Figure 9 illustrates a map of $F S_{\text {Triggering }}$ inside the structure at 18 th second of earthquake excitation thus practically after it has finished. Despite short epicenter distance, liquefaction does not penetrate into embankment core, influencing only the soil on the base of both slopes along with isolated local areas on the slope edges. 


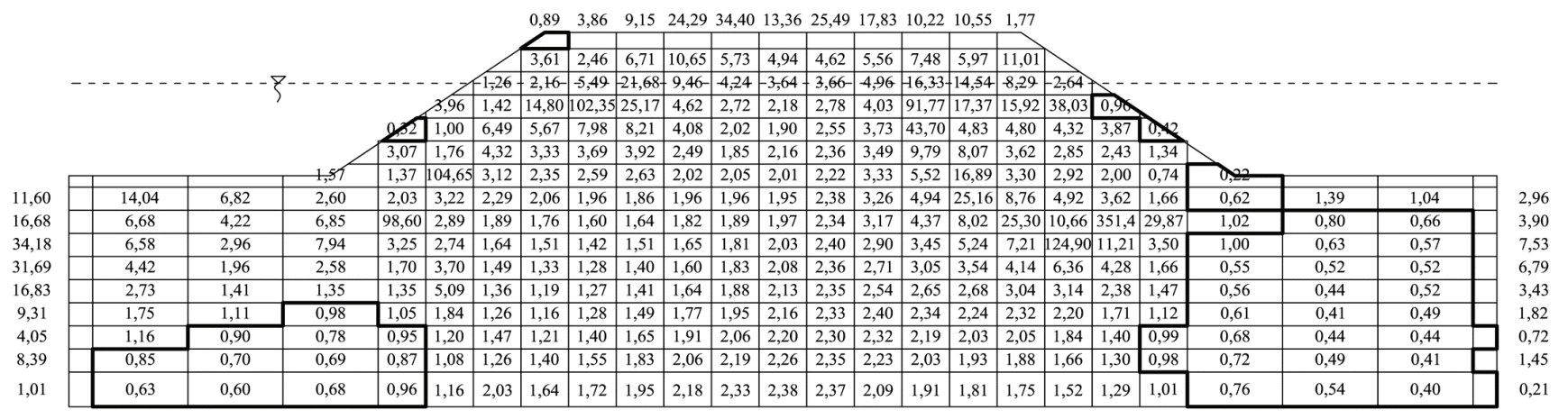

Fig. 9. Factor of safety distribution inside embankment for the earthquake excitation (WTL at $6 \mathrm{~m}$ )

\section{CONCLUSIONS}

For the modeled structure, cyclic point loads (i.e., vibrating roller) present higher risk than earthquake of magnitude 5.4. This problem will require in the future a further analysis because, as geotechnical practice shows, dams can be damaged or destroyed by the earthquakes of comparable magnitudes (see San Fernando Dam failure). Why the structure considered is less susceptible to earthquake than typical dam could be explained by stabilizing and damping influence of water, acting here on both sides of the slope.

As the origin factor of safety against triggering liquefaction is calculated not for maximum shear stresses but for the peak values of pore pressures, the modified factor was established. It showed closed failure of the subsoil and the increase of sensitivity to the effects of dynamic actions, even at large distances from the source of vibration.

The method introduced in the paper presents a clear and easy way to locate liquefied zones and estimate liquefaction susceptibility of the subsoil - not only in the road embankment. Thus, it could be very helpful in geotechnical practice, as long as the stresses are obtained from programs (like Plaxis 2D) based on Biot's theory of dynamic consolidation [7].

\section{REFERENCES}

[1] Jeffries M., BeEn K., Soil Liquefaction. A critical state approach, Taylor \& Francis, 2006.

[2] Das B.M., Principles of Soil Dynamics, Pacific Grove, Brooks/Cole, 1993.

[3] Borowiec A., Wrana B., 2007, Transformaty falkowe $w$ dynamice gruntów, Czasopismo Techniczne Politechniki Krakowskiej, z. 6-B, 2007, 107-114.

[4] Schanz T., Vermeer PA., Bonnier PG., The hardening soil model: Formulation and verification, Beyond 2000 in Computational Geotechnics, Balkema, Rotterdam, 1999, 281-290.

[5] Tiznado J.C., Rodriguez-RoA F., Seismic lateral movement prediction for gravity retaining walls on granular soils, Soil Dynamics and Earthquake Engineering, Elsevier, Oxford, 2011, 31, 391-400.

[6] Olson S.M., Stark T.D., Yield Strength Ratio and Liquefaction Analysis of Slopes and Embankments, Journal of Geotechnical and Geoenvironmental Engineering, ASCE, 2003, 129(8), 727-737.

[7] Plaxis 2D Manuals, 2010, electronic documentations. 